\title{
Methadone-A Replacement for Deadly Heroin, Can Kill you too!!
}

\section{Gurveen Malhotra $^{1 *}$ and Sivashankar Sivaraman ${ }^{2}$}

${ }^{1}$ Internal Medicine, Mt Sinai Hospital, Chicago, USA

${ }^{2}$ Pulmonary and Critical Care, Mt Sinai Hospital, Chicago, USA

\begin{abstract}
Methadone is widely used as a replacement for heroin addiction and an inexpensive treatment for chronic pain. We report the case of a 51 year old male on high dose methadone treatment for heroin addiction who presented with prolonged QT interval on electrocardiogram and later had a cardiac arrest with Torsades de Pointes (TdP), a rare complication.
\end{abstract}

Keywords: Methadone; Prolonged QT; Torsades de Pointes; Cardiac arrest

\section{Case Presentation}

A 51 year old male with history of hepatitis $\mathrm{C}$, liver cirrhosis and on methadone treatment for heroin addiction presented to the emergency room with lethargy, weakness and acute confusion. He was taking $170 \mathrm{mg}$ methadone daily and lorazepam only occasionally for anxiety. He however was still abusing heroin and had an alcohol problem as well. As per the significant other, patient was having brief lapses in consciousness for 2 days associated with sweating unrelated to postural changes. They were not associated with preceding chest pain, dizziness, palpitations or seizure like activity.

He was hemodynamically stable except for blood pressure elevated to $160 / 75 \mathrm{~mm} \mathrm{Hg}$ with disorientation to time. Neurological exam did not reveal any focal neurological deficits or meningeal signs. Other pertinent findings were stigmata of liver cirrhosis with ascites but no asterixis or abdominal tenderness.

Electrocardiogram was remarkable for corrected QT interval prolonged to $605 \mathrm{msec}$ and ventricular bigeminy (Figure 1) without

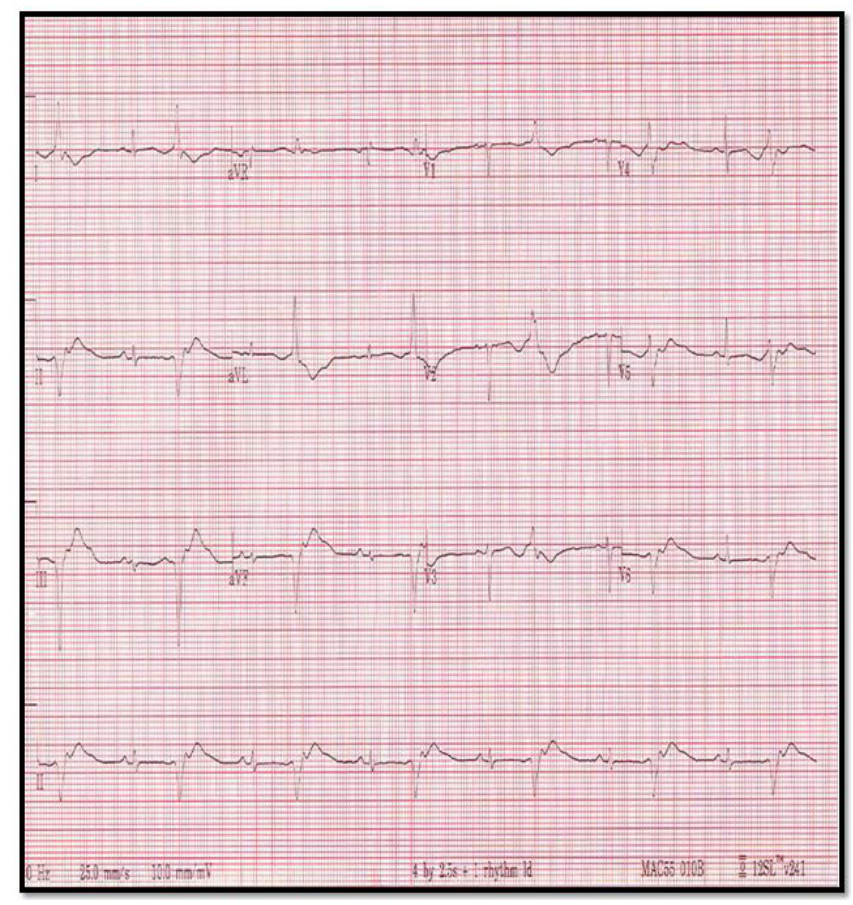

Figure 1: Electrocardigram.

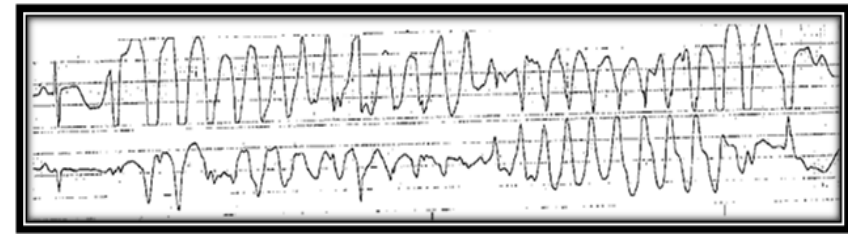

Figure 2: Rhythm strips.

any atrioventricular block. Blood work revealed hemoglobin of 10.5, platelet count 125 , serum creatinine 2.02 , potassium 4.2 and magnesium $1.3(1.3-2.1 \mathrm{meq} / \mathrm{L})$. Arterial blood gas did not show carbon dioxide retention and blood ammonia level was normal at $39(16-53 \mu \mathrm{mol} / \mathrm{L})$. A computerized tomography scan of the head did not reveal any acute intracranial abnormalities.

Magnesium sulfate infusion was given and on the night of presentation, he had a cardiac arrest with $\mathrm{TdP}$ (Figure 2) as the presenting rhythm. He was successfully resuscitated after a prolonged cardiac arrest of 17 minutes involving defibrillation. Magnesium was given intravenously and he was transferred to the intensive care unit. Patient was neurologically stable after the arrest and did not require hypothermia protocol. After the cardiac arrest, he developed sinus bradycardia to low $50 \mathrm{~s}$ requiring isoproterenol drip to prevent recurrence of TdP. The target heart rate was around 90-100 per minute. Echocardiogram showed stage 11 diastolic dysfunction with moderately dilated left atrium but no regional wall motion abnormalities. QT interval continued to be prolonged to as high as $700 \mathrm{msec}$ and eventually started becoming shorter. Patient showed a good positive chronotropic response to isoproterenol and did not require overdrive pacing. Methadone was held all this time. The isoproterenol drip was slowly tapered off and the QT normalized to around $400 \mathrm{msec}$ over the next few days. No further episodes of TdP were seen during the hospitalization.

\section{Discussion}

Methadone has a serious side effect of prolonging QT interval. It does so by inhibiting the rapid component of delayed rectifier

*Corresponding author: Gurveen Malhotra, PGY-3, Department of Internal Medicine, Mt Sinai Hospital, Chicago, USA, Tel: 773-610-8973; E-mail: gurveen22@gmail.com

Received April 20, 2015; Accepted June 22, 2015; Published June 26, 2015

Citation: Malhotra G, Sivaraman S (2015) Methadone-A Replacement for Deadly Heroin, Can Kill you too!! J Clin Case Rep 5: 551. doi:10.4172/2165-7920.1000551

Copyright: (c) 2015 Malhotra G, et al. This is an open-access article distributed under the terms of the Creative Commons Attribution License, which permits unrestricted use, distribution, and reproduction in any medium, provided the original author and source are credited. 
potassium ion current $[1,2]$. Prolongation of QT may lead to TdP which is a polymorphic ventricular tachycardia. Methadone-induced TdP is a rare complication and the true incidence is unknown. One study estimated the incidence to be less than $1 \%$ while others showed it to be around 3\% [3-5].

We suspect hypomagnesemia in the setting of alcohol abuse, liver cirrhosis and high dose methadone use precipitated TdP in our patient. Further hepatitis $\mathrm{C}$ infection has also been seen to independently cause significant QT prolongation [6].

The acute management is intravenous magnesium administration [7] and holding the offending agent. Magnesium sulfate 2 grams can be infused intravenously irrespective of the serum magnesium level [8].

Bradycardia may predispose to TdP [9] and requires treatment with positive chronotropic agents like isoproterenol and overdrive pacing in patients with heart block, symptomatic bradycardia or recurrent pause dependent TdP [7].

\section{References}

1. Katchman AN, Ebert SN, McGroary KA, Woosley RL (2001) Methadone blocks HERG current in transfected HEK cells. The Pharmacologist 43: 98.

2. Rendig SV, Amsterdam EA, Henderson GL, Mason DT (1980) Comparative cardiac contractile actions of six narcotic analgesics: morphine, meperidine, pentazocine, fentanyl, methadone and I alphaacetylmethadol (LAAM). J Pharmacol Exp Ther 215: 259-265.

3. Sticherling C, Schaer BA, Ammann P, Maeder M, Osswald S (2005) Methadoneinduced TdP tachycardias. Swiss Med Wkly 135: 282-285.

4. Ehret GB, Voide C, Gex-Fabry M, Chabert J, Shah D, et al. (2006) Druginduced long QT syndrome in injection drug users receiving methadone. Arch Intern Med 166: 1280-1287.

5. George S, Moreira K, Fapohunda M (2008) Methadone and the heart: what the clinician needs to know. Curr Drug Abuse Rev 1: 297-302.

6. Nordin C, Kohli A, Beca S, Zaharia V, Grant T, et al. (2006) Importance of hepatitis $\mathrm{C}$ coinfection in the development of QT prolongation in HIV-infected patients. J Electrocardiol 39: 199-205.

7. Zipes DP, Camm AJ, Borggrefe M, Buxton AE, Chaitman B (2006) ACC/AHA/ ESC 2006 guidelines for management of patients with ventricular arrhythmias and the prevention of sudden cardiac death: a report of the American College of Cardiology/American Heart Association Task Force and the European Society of Cardiology Committee for Practice Guidelines (Writing Committee to Develop Guidelines for Management of Patients With Ventricular Arrhythmias and the Prevention of Sudden Cardiac Death). J Am Coll Cardiol 48: e247-346.

8. Banai S, Tzivoni D (1993) Drug therapy for torsade de pointes. J Cardiovasc Electrophysiol 4: 206-210.

9. Díaz-Castro O, Puchol A, Almendral J, Torrecilla EG, Arenal A, et al. (2004) Predictors of in-hospital ventricular fibrillation or torsades de pointes in patients with acute symptomatic bradycardia. J Electrocardiol 37: 55-60. 